\title{
Predictive Effects of Circulating miR-221, miR-130a and miR-155 for Coronary Heart Disease: A Multi-Ethnic Study in China
}

\author{
Qiao-Wei Jia ${ }^{a}$ Zhao-Hong Chen ${ }^{b} \quad$ Xiao-Qing Ding ${ }^{a}$ Jie-Yin Liu ${ }^{a}$ Peng-Cheng Ge ${ }^{a}$ \\ Feng-Hui An ${ }^{b}$ Li-Hua Lib Lian-Sheng Wang ${ }^{a}$ Wen-Zhu Ma ${ }^{a}$ Zhi-Jian Yanga \\ En-Zhi Jia ${ }^{a}$
}

aDepartment of Cardiovascular Medicine, the First Affiliated Hospital of Nanjing Medical University, Nanjing, bDepartment of Cardiovascular Medicine, the Friendship Hospital of Ili Kazakh Autonomous Prefecture, Yining, China

\section{Key Words}

MicroRNA • Coronary heart disease • Multi-ethnicity

\begin{abstract}
Background: Differences in microRNA (miRNA) profiles between patients with and without coronary heart disease (CHD)have not been fully determined. The purpose of the study was to evaluate in a multi-ethnic population in China the predictive value of miRNAs previously suggested to have a role in CHD. Subject and method: 932 participants were included, and plasma samples obtained. A quantitative reverse-transcription PCR(RT-qPCR) assay was conducted to confirm the concentration of plasma miRNAs. Circulating levels of miRNAs were quantified using the $2^{-\Delta c t}$ method. The severity of coronary atherosclerosis was evaluated via Gensini Scores. Result: The circulating levels of the nine proposed miRNAs were not different among the five main ethnicities examined (all $p>0.05$ ). The Spearman correlation analyses indicated that miR-221 and miR-130a were negatively associated with the severity of CHD as indicated by Gensini Scores $(r=-0.106, p=0.001 ; r=-0.073, p=0.026)$. Results of the univariate analysis showed that lower circulating miR-221 (OR, 1.663; $95 \% \mathrm{Cl}, 1.255-2.202, p$ $=<0.001)$, miR-155 (OR, 1.520; $95 \%$ Cl, 1.132-2.042, $p=0.005)$, and miR-130a (OR, 1.943; 95 $\% \mathrm{Cl}, 1.410-2.678, p=<0.001)$ were potential risk factors for CHD. Moreover, miR-130a (OR, 2.405; $95 \% \mathrm{Cl}, 1.691-3.421, p=<0.001)$ remained independently associated with the risk of CHD after adjusting for potential confounding factors. The analysis of the possible positive/ negative associations between miR-221, miR-155 and miR-130awere conducted. A positive association between miR-130a and miR-155 was found $(S I=1.60, S I M=1.21$ and $A P=0.22)$, and in these groups, the proportion of CHD attributable to the interaction between miR-130a and miR-155 was as high as $22 \%$. A negative interaction was found between miR-221 and miR-130a $(S I=0.68, S I M=0.60$ and $A P=0.27)$. Conclusion: Plasma levels of miR-221, miR$130 \mathrm{a}$ and miR-155 decreased in patients with CHD, and miR-130a may be an independent predictor for CHD.

Q. Jia and F. An contribued to the work equally.

(C) 2017 The Author(s)

Published by S. Karger AG, Basel

Dr. En-Zhi Jia

Department of Cardiovascular Medicine, the First Affiliated Hospital of Nanjing Medical University, Guangzhou Road 300, Nanjing 210029, Jiangsu Province (China)

Tel. +86-13951623205, Fax 0086-025-84352775, E-Mail enzhijia@njmu.edu.cn
\end{abstract}




\section{Cellular Physiology Cell Physiol Biochem 2017;42:808-823 and Biochemistry Published onIne: June 19, $2017 \quad$\begin{tabular}{l|l} 
DOI: 10.1159/000478071 & $\begin{array}{l}\text { (c) } 2017 \text { The Author(s). Published by S. Karger AG, Basel } \\
\text { www.karger.com/cpb }\end{array}$
\end{tabular} Jia et al.: Potential Biomarkers for Detection of CHD}

\section{Introduction}

Despite significant improvements in diagnosis and treatment in recent decades, cardiovascular diseases (CVD), particularly coronary heart disease (CHD), remain the leading cause of human morbidity and mortality in the world [1]. Data from the World Health Organization (WHO) shows that approximately 17.5 million people died from CVDs in 2012 (7.4 million caused by CHD), which accounts for $31 \%$ of all global deaths [2]. In China, the age-standardized mortality of CVD in 2002 was 300 persons per 100,000 [3]. Early prevention of CHD is of clinical significance for the overall reduction of CVD mortality. Although significant advancement has been achieved for the early prediction of acute CVD, such as acute coronary syndrome, biomarkers for the detection chronic stable CHD remains a challenge in clinical practice.

CHD is caused by coronary atherosclerosis, an inflammatory disease due to the formation of plaque and subsequent obstruction of the coronary arteries. It has been suggested that during the process of plaque formation, the cellular components of the plaque may release miRNAs into the circulation. These have the potential to serve as new biomarkers for predicting CHD [4-5]. miRNAs are endogenous, small non-coding RNAs which are $\sim 22$ nucleotides in length, and are involved in the post-transcriptional regulation of genes. Functional changes, including up-regulation and down-regulation of miRNAsare known to be involved in the pathogenesis of many diseases such as myocardial infarction [6-9], coronary artery calcification (CAC) [10], arrhythmia [11], cardiomyocyte hypertrophy [12-13], heart failure [14], renal ischemia-reperfusion injury [15], non-alcoholic fatty liver disease [16], tuberculosis [17], pancreatic ductal adenocarcinoma [18], and diabetes mellitus [19]. miRNAs are reported to not only exist within the cell but also reside stably in the extracellular compartments such as the circulation, which enables relatively simple detection of miRNAs as disease biomarkers. Another interesting characteristic of diseasespecific miRNAs is that they may be ethnicity specific [20]. Moreover, accumulating evidence indicates that some miRNAs may be specific for patients with CHD [21]. However, the potential role of these miRNAs in multi-ethnic CHD patients has rarely been reported. In this study, we aimed to evaluate the predictive value of previously suggested miRNAs for CHD in a multi-ethnic population in China.

\section{Subject and Methods}

\section{Study subjects}

From March 1, 2010 to April 31, 2015, 932 consecutive adult subjects (681 males and 251 females) aged 32-84 years, who underwent coronary angiography for suspected or known coronary atherosclerosis at the Friendship Hospital of Ili Kazakh Autonomous Prefecture in China, were selected in this study. Among the 932 subjects, 587 subjects were of Han ethnicity, 146 were of Uygur ethnicity, 91 were of Kazakh ethnicity, 67 were of Hui ethnicity, and 41 were of other ethnicities ( 5 were of Mongol ethnicity, 26 were of Siwe ethnicity, 2 were of Kyrgyz ethnicity, 2 were of Uzbeks ethnicity, 2 were of Manju ethnicity, 2 were of Daur ethnicity, and 2 were of Russian ethnicity).Subjects with spastic angina pectoris, infectious processes within 2 weeks, heart failure, adrenal dysfunction, and thyroid dysfunction were excluded. The study was performed in accordance with approved guidelines, and all experimental protocols were approved by the ethics committee of the First Affiliated Hospital of Nanjing Medical University and the Friendship Hospital of Ili Kazakh Autonomous Prefecture in China. All subjects provided written informed consent.

\section{Coronary Angiography}

Coronary arteries were cannulated using either the Judkins technique [22] or through a radial artery approach with $6 \mathrm{~F}$ catheters and recorded at a rate of 30 frames/s. Significant CHD was defined as at least one major epicardial vessel with $>50 \%$ stenosis; control subjects were defined as all of the major epicardial vessels with $<50 \%$ stenosis [23]. The severity of the coronary atherosclerosis was based on the Gensini scoring system [24-27]. 


\section{Cellular Physiology Cell Physiol Biochem 2017;42:808-823 \begin{tabular}{l|l} 
and Biochemistry Published 10.1159/000478071 & $\begin{array}{l}\text { (c) 2017 The Author(s). Published by S. Karger AG, Basel } \\
\text { www.karger.com/cpb }\end{array}$
\end{tabular}}

Jia et al.: Potential Biomarkers for Detection of CHD

\section{Laboratory measurements}

Four millilitres of venous blood was drawn after 12 hours of fasting to perform biochemical assays on the second day of hospitalization; total cholesterol (TCH, mmol/L), triglyceride (TG, $\mathrm{mmol} / \mathrm{L}$ ), fasting blood glucose (FBG, mmol/L), creatinephosphokinase-myoglobin isoenzyme(CK-MB, U/L), fasting highdensity lipoprotein cholesterol (HDL-C, mmol/L), fasting low-density lipoprotein cholesterol (LDL-C, mmol/L), apolipoprotein A (Apo-A, g/L) and apolipoprotein B (Apo-B, g/L) were determined by enzymatic procedures on an automated autoanalyzer (AU 2700 Olympus, 1st Chemical Ltd, Japan). Excellent intraassay and inter-assay CVs of $<5 \%$ were obtained with our assay method.

\section{Selection of miRNAs}

Based on previous studies [28], nine CHD-related miRNAs were proposed as candidates in the study. This includedmiR-122, miR-133b [29], miR-214 [30], miR-21, miR-106a [31], miR-130a, miR-155, miR-221 [32], and miR-125b [33-36].

\section{Plasma preparation and RNA isolation}

All samples $(5 \mathrm{ml})$ were collected in EDTA plasma tubes on the morning following arrival. Samples were processed within 4 hours and stored at $4{ }^{\circ} \mathrm{C}$. Plasma was collected after centrifugation $(15 \mathrm{~min}$ at $1000 \times \mathrm{g}$ ) and was stored at $-80^{\circ} \mathrm{C}$ until further analysis.

For the RT-qPCR assay of plasma, total RNA was extracted using the one-step phenol/chloroform purification protocol, as previously reported [37].

\section{Quantification of miRNAs by RT-qPCR analysis}

For miRNA profiling, the RT-qPCR assay was performed using a TaqMan PCR kit according to the manufacturer's instructions (Applied Biosystems, Foster City, USA); a minor modification was made according to the State Key Laboratory of Pharmaceutical Biotechnology (School of Life Sciences, Nanjing University), reported in 2010 [38]. Due to the superior performance of a combination of let-7d, let-7g and let-7i, this combination was chosen as a reference for the normalization of plasma miRNAs rather than the commonly used reference genes U6, RNU44, RNU48 and miR-16 [39]. The resulting threshold cycle (CT) values were determined according to the default threshold settings when the reactions were complete. The relative amount of each miRNA was calculated based on the internal control (i.e., the combination of let-7d, let-7g and let-7i, analysed using the $2^{-\Delta c t}$ method, which is a widely used method for presenting relative gene expression by comparative CT). The calculation formula was as following: 2exp - (mean CT target miRNA mean CT internal control) [40-41] for CT $<40$ and $2^{-40}$ in the case $\mathrm{Ct} \geq 40$ (considered as undetermined) [42]. The rate of undetermined miRNAs (CT >40) was as following: $47.1 \%$ for miR-125b, $26.7 \%$ for miR-122, $49.1 \%$ for miR-214, $38.5 \%$ for miR-133b, $10.8 \%$ for miR-221, $8.3 \%$ for miR-21, $8.2 \%$ for miR-155, $8.9 \%$ for miR-106a, and $8.0 \%$ for miR-130a.

\section{Gene Ontology and Pathway analysis}

Gene Ontology (GO) functional classification was performed byBlast2GO software (http://www. blast2go.de), and contigs were divided into biological processes, cellular components and molecular functions according to GO terms.GO analysis was used to analyze the main function of differently expressed genes according to GO, which is the key functional classification of NCBI. This classification system organizes genes into hierarchical categories and identifies the gene regulatory network based on biological process, cellular components and molecular functions. A two-tailed Fisher's exact test and $X^{2}$ test were used to classify the GO category, and the FDR was calculated to correct the $P$ value. A smaller FDR indicated a smaller error in judging the $P$ value.Pathway analysis was used to determine significant pathways based on differential gene expression according to the KEGG. Fisher's exact test was used to select the significant pathway, and the threshold of significance was defined by the $P$ value and FDR.

\section{Statistical analysis}

Data were statistically analysed using Statistics Package for Social Sciences (ver. 16.0; SPSS Incorporated, Chicago, IL, USA). Subjects were classified into five groups according to ethnicity, two groups according to CHD status and four groups according to the quartile of the Gensini score. Data for age, TG, TCH, HDL-C, LDL-C, FBG, CK-MB, Apo-A, Apo-B, Gensini scores, miR-122, miR-133b, miR-214, miR-21, 


\section{Cellular Physiology Cell Physiol Biochem 2017;42:808-823 and Biochemistry Published onlıne: June 19, $2017 \quad \begin{aligned} & \text { DOI: 10.1159/000478071 } 2017 \text { The Author(s). Published by S. Karger AG, Basel } \\ & \text { www.karger.com/cpb }\end{aligned}$ \\ Jia et al.: Potential Biomarkers for Detection of CHD}

miR-106a, miR-130a, miR-155, miR-221, and miR-125b were skewed distributed parameters and were expressed as median and quartile ranges, with comparisons performed using the Mann-Whitney U test and Kruskal-Wallis $\mathrm{H}$ test. The categorical variable of gender was compared between or among the groups of patients using a chi-squared analysis. The Spearman two-way test was used to assess the relationship between Gensini scores with miRNAs and classical risk factors. The receiver operating characteristic (ROC) curve was performed, and the area under the ROC curve $(A U C)$ was calculated to evaluate the specificity and sensitivity of CHD prediction for each miRNA [43]. ORs regarding the presence of obstructive CHD were determined via either a univariate or a multivariate logistic regression analysis and are presented with 95 $\%$ CIs. Differences were considered to be significant if the null hypothesis could be rejected with > $95 \%$ confidence. All $P$-values were two-tailed.To further explore the synergistic effect of miRNA on the risk of CHD, a $4 \times 2$ table approach was conducted to calculate odds ratios (ORs), $95 \%$ confidence intervals (CIs) and two-tailed $P$-values, and multiple indexes (SI, SIM, RERI, and $A P$ ) were used to evaluate the synergistic effect between miRNAs [44, 45].

\section{Results}

\section{Baseline characteristics of subjects grouped according to ethnicity}

A total of 932 subjects (681 males and 251 females) with a median age of 60-years were selected in the study. The baseline characteristics of the subjects grouped according to ethnicity are presented in Table 1 . Significant differences in age $(p=0.020)$, CK-MB $(p=$ $<0.001)$, LDL-C ( $p=0.003)$, apolipoprotein A ( $p=0.007)$, apolipoprotein B ( $p=0.018)$, and FBG ( $p=0.028)$ were observed among the various ethnicities, with the frequency distribution in CHD status $(p=0.012)$ significantly differing among the various ethnicities. However, there was no statistical difference in gender $(p=0.063), \mathrm{TCH}(p=0.093)$, triglyceride $(p=$ $0.067), \mathrm{HDL}-c(p=0.337), \operatorname{miR}-125 \mathrm{~b}(p=0.561), \operatorname{miR}-122(p=0.589), \operatorname{miR}-214(p=0.197)$, $\operatorname{miR}-133 b(p=0.486), \operatorname{miR}-221(p=0.737), \operatorname{miR}-21(p=0.410), \operatorname{miR}-155(p=0.783)$, miR106a ( $p=0.112)$, and miR-130a ( $p=0.714)$ among the various ethnicities.

\section{Demographic, clinical and biochemical characteristics of subjects according to Gensini} Score

Gensini Score of the subjects in the present study ranged from 0 to 240.00 , with a median of 16.00 (quartile range, 3.00-45.00). Table 2 shows the demographic, clinical and

Table 1. Baseline characteristics of the subjects grouped according to ethnicity. Data are summarized by 50 th (25th/75th) percentiles for continuous variables and $N_{1} / N_{2}$ for binary variables. CK-MB, MB isoenzyme of creatine kinase; TCH, total cholesterol; TG, triglyceride; HDL-C, fasting high-density lipoprotein cholesterol; LDL-C, fasting low-density lipoprotein cholesterol; Apo-A, apolipoprotein A; Apo-B, apolipoprotein A; FBG, fasting blood glucose. The value of each miRNA means the relative amount calculated by $2^{-\Delta c t}$ method

\begin{tabular}{|c|c|c|c|c|c|c|c|}
\hline Variable & $\begin{array}{c}\text { Han } \\
(n=587)\end{array}$ & $\begin{array}{c}\text { Hui } \\
(n=67)\end{array}$ & $\begin{array}{c}\text { nicity }(\mathrm{n}=932) \\
\text { Uygur } \\
{[\mathrm{n}=146]}\end{array}$ & $\begin{array}{l}\text { Kazakh } \\
(\mathrm{n}=91)\end{array}$ & $\begin{array}{c}\text { Others } \\
{[n=41]}\end{array}$ & $\begin{array}{l}\text { F or chi- } \\
\text { square test }\end{array}$ & $P$ \\
\hline Age (years) & $62.00(52.00-70.00)$ & $58.00(54.00-64.00)$ & $60(53.75-66.25)$ & $57.00(50.00-64.00)$ & $64.00(54.50-68.50)$ & 11.727 & 0.020 \\
\hline Gender (M/F) & $414 / 173$ & $55 / 12$ & $108 / 38$ & $75 / 16$ & $29 / 12$ & 8912 & 0.063 \\
\hline CK-MB (U/L) & $16.00(12.50-21.00)$ & $16.00(13.40-22.00)$ & $18.00(14.00-24.25)$ & $19.00(15.00-24.00)$ & $16.00(12.50-21.50)$ & 25.227 & $<0.001$ \\
\hline $\mathrm{TCH}(\mathrm{mmol} / \mathrm{L})$ & $4.61(3.90-5.45)$ & $4.35(3.58-5.33)$ & $4.88(4.09-5.69)$ & $4.61(3.80-528)$ & $4.72(3.91-5.48)$ & 7957 & 0.093 \\
\hline $\mathrm{TG}(\mathrm{mmol} / \mathrm{L})$ & $182(1.25-248)$ & $1.72(1.08-253)$ & $1.71(1.242 .43)$ & $1.52(1.07-2.05)$ & $203(1.26-277)$ & 8.775 & 0.067 \\
\hline $\mathrm{HDL}-\mathrm{C}(\mathrm{mmol} / \mathrm{L})$ & $136(1.14-1.62)$ & $1.32(1.14-1.61)$ & $1.46(1.16-1.70)$ & $129(1.06-1.62)$ & $139(1.17-1.60)$ & 4550 & 0.337 \\
\hline LDL-C (mmol/L) & $2.68(2.14-3.31)$ & $2.68(1.97-3.54)$ & $3.05(2.45-3.86)$ & $285(2.22-3.53)$ & $2.96(2.07-3.42)$ & 16.331 & 0.003 \\
\hline Apo-A $(g / L)$ & $1.29(1.17-1.46)$ & $1.33(1.19-1.51)$ & $1.25(1.12-1.42)$ & $125(1.10-1.38)$ & $1.20(1.10-1.48)$ & 14.240 & 0.007 \\
\hline Apo-B (g/L) & $0.89(0.76-1.04)$ & $0.87(0.71-1.06)$ & 0.97 (080-1.12) & $0.89(0.70-1.05)$ & $0.87(0.74-1.00)$ & 11870 & 0.018 \\
\hline $\mathrm{FBG}(\mathrm{mmol} / \mathrm{L})$ & $5.07(4.62-6.03)$ & $521(4.75-7.07)$ & $5.08(4.56-5.94)$ & $4.95(4.49-5.55)$ & $4.97(4.76-5.55)$ & 10.891 & 0.028 \\
\hline miR-125b & $0.04(0.00-0.24)$ & $0.00(0.00-0.18)$ & $0.04(0.00-0.21)$ & $0.02(0.00-028)$ & $0.15(0.00-0.27)$ & 2982 & 0.561 \\
\hline miR-122 & $0.15(0.00-1.77)$ & $027(0.01-2.17)$ & $0.29(0.00-1.85)$ & $031(0.00-1.54)$ & $1.00(0.02-1.88)$ & 2815 & 0.589 \\
\hline miR-214 & $0.00(0.00-0.23)$ & $0.01(0.00-0.28)$ & $0.00(0.00-0.22)$ & $0.00(0.00-026)$ & $0.13(0.00-0.36)$ & 6.023 & 0.197 \\
\hline $\mathrm{miR}-133 \mathrm{~b}$ & $0.17(0.00-0.44)$ & $0.16(0.00-0.49)$ & $0.20(0.00-0.53)$ & $0.14(0.00-0.41)$ & $0.15(0.00-0.42)$ & 3.444 & 0.486 \\
\hline miR-221 & $0.10(0.03-0.18)$ & $0.09(0.05-0.24)$ & $0.09(0.03-0.17)$ & $0.11(0.02-022)$ & $0.10(0.04-0.30)$ & 1.992 & 0.737 \\
\hline miR-21 & $182(0.62-4.60)$ & $184(0.75-623)$ & $2.06(0.64-4.51)$ & $2.13(0.44-5.94)$ & $0.93(0.46-3.65)$ & 3.973 & 0.410 \\
\hline miR-155 & $200.25(5021-3350.13)$ & $188.71(74.03-2368.90)$ & $150.18(42.44-2138.68)$ & $121.94(45.89-1686.71)$ & $158.68(40.79-297156)$ & 1.740 & 0.783 \\
\hline miR-106a & $1.61(0.83-3.16)$ & $1.65(0.95-3.36)$ & $1.48(0.68-2.47)$ & $128(0.62-2.77)$ & $1.22(0.78-182)$ & 7.492 & 0.112 \\
\hline miR-130a & $3.18(1.56-5.70)$ & $3.43(1.52-13.93)$ & $2.93(1.41-5.26)$ & $2.99(1.06-5.94)$ & $3.43(1.62-12.96)$ & 2.116 & 0.714 \\
\hline
\end{tabular}


Table 2. Characteristics of the study population categorized by the quartile of Gensini Scores: results of $\mathrm{K}$ independent sample test. CK-MB, MB isoenzyme of creatine kinase; TCH, total cholesterol; TG, triglyceride; HDL-C, fasting high-density lipoprotein cholesterol; LDL-C, fasting low-density lipoprotein cholesterol; ApoA, apolipoprotein A; Apo-B, apolipoprotein A; FBG, fasting blood glucose. The value of each miRNA means the relative amount calculated by $2^{-\Delta c t}$ method

\begin{tabular}{|c|c|c|c|c|c|c|}
\hline \multirow{2}{*}{ Characteristics } & \multicolumn{4}{|c|}{ Gensini Scores } & \multirow{2}{*}{$\begin{array}{l}\text { Statistical } \\
\text { parameter }\end{array}$} & \multirow{2}{*}{$P$ value } \\
\hline & $0(\mathrm{n}=189)$ & $0.1-3.0(n=63)$ & $3.5-44(n=445)$ & $44.5(n=235)$ & & \\
\hline Age (years) & $57.00(49.00-65.00)$ & $58.00(49.00-68.00)$ & $62.00(54.00-70.00)$ & $62.00(55.00-69.00)$ & 30.634 & $<0.001$ \\
\hline Gender $(\mathrm{M} / \mathrm{F})$ & $118 / 71$ & $48 / 15$ & $330 / 115$ & $185 / 50$ & 15.261 & 0.002 \\
\hline CK-MB (U/L) & $16.00(13.00-20.00)$ & $17.00(14.00-20.00)$ & $16.00(13.00-21.00)$ & $18.00(13.00-26.00)$ & 11.453 & 0.010 \\
\hline $\mathrm{TCH}(\mathrm{mmol} / \mathrm{L})$ & $4.58(3.91-5.27)$ & $4.47(3.60-5.22)$ & $4.66(3.90-5.50)$ & $4.69(3.96-5.67)$ & 5.124 & 0.163 \\
\hline $\mathrm{TG}(\mathrm{mmol} / \mathrm{L})$ & $1.71(1.14-2.36)$ & $1.59(1.13-2.09)$ & $1.80(1.26-2.52)$ & $1.83(1.24-2.50)$ & 5.727 & 0.126 \\
\hline $\mathrm{HDL}-\mathrm{C}(\mathrm{mmol} / \mathrm{L})$ & $1.41(1.20-1.64)$ & $1.35(1.17-1.64)$ & $1.36(1.12-1.62)$ & $1.32(1.08-1.66)$ & 4.941 & 0.176 \\
\hline $\mathrm{LDL}-\mathrm{C}(\mathrm{mmol} / \mathrm{L})$ & $2.71(2.16-3.38)$ & $2.62(1.98-3.23)$ & $2.74(2.15-3.42)$ & $2.90(2.26-3.55)$ & 8.548 & 0.036 \\
\hline Apo-A $(\mathrm{g} / \mathrm{L})$ & $1.30(1.18-1.48)$ & $1.35(1.18-1.47)$ & $1.29(1.15-1.46)$ & $1.25(1.11-1.41)$ & 12.639 & 0.005 \\
\hline Apo-B (g/L) & $0.89(0.77-1.03)$ & $0.87(0.68-1.02)$ & $0.90(0.74-1.07)$ & $0.91(0.77-1.06)$ & 2.505 & 0.474 \\
\hline $\mathrm{FBG}(\mathrm{mmol} / \mathrm{L})$ & $4.84(4.50-5.23)$ & $5.00(4.72-5.50)$ & $5.13(4.63-6.04)$ & $5.34(4.62-6.37)$ & 29.441 & $<0.001$ \\
\hline miR-125b & $0.01(0.00-0.23)$ & $0.15(0.00-0.32)$ & $0.04(0.00-0.25)$ & $0.02(0.00-0.20)$ & 5.220 & 0.156 \\
\hline miR-122 & $0.28(0.01-1.35)$ & $0.48(0.01-2.83)$ & $0.13(0.00-1.69)$ & $0.35(0.00-1.95)$ & 4.782 & 0.188 \\
\hline miR-214 & $0.01(0.00-0.26)$ & $0.05(0.00-0.29)$ & $0.00(0.00-0.23)$ & $0.00(0.00-0.23)$ & 3.809 & 0.283 \\
\hline miR-133b & $0.16(0.00-0.40)$ & $0.28(0.00-0.58)$ & $0.17(0.00-0.46)$ & $0.14(0.00-0.48)$ & 4.742 & 0.192 \\
\hline miR-221 & $0.12(0.04-0.26)$ & $0.11(0.05-0.30)$ & $0.09(0.03-0.17)$ & $0.08(0.03-0.16)$ & 14.597 & 0.002 \\
\hline miR-21 & $1.72(0.61-4.48)$ & $2.00(0.54-6.15)$ & $1.93(0.64-4.53)$ & $1.78(0.55-4.76)$ & 0.283 & 0.963 \\
\hline miR-155 & $252.48(64.22-3395.90)$ & $205.07(72.00-382.68)$ & $149.09(45.89-1944.54)$ & $190.02(39.95-4182.07)$ & 4.490 & 0.213 \\
\hline miR-106a & $1.61(0.87-3.34)$ & $1.62(0.92-3.20)$ & $1.44(0.71-3.08)$ & $1.52(0.80-2.66)$ & 3.637 & 0.303 \\
\hline miR-130a & $3.61(1.70-16.77)$ & $2.95(2.01-7.84)$ & $3.10(1.42-5.52)$ & $2.95(1.49-5.21)$ & 8.194 & 0.042 \\
\hline
\end{tabular}

Table 3. Spearman correlations between Gensini Scores and features of the study population. CK-MB, MB isoenzyme of creatine kinase; TCH, total cholesterol; TG, triglyceride; HDL-C, fasting high-density lipoprotein cholesterol; LDL-C, fasting low-density lipoprotein cholesterol; Apo-A, apolipoprotein A; Apo-B, apolipoprotein A; FBG, fasting blood glucose. The value of each miRNA means the relative amount calculated by $2^{-\Delta c t}$ method

\begin{tabular}{lcc}
\hline Variables & Relationship coefficient & $P$ value \\
\hline Age (years) & 0.152 & $<0.001$ \\
CK-MB (U/L) & 0.144 & $<0.001$ \\
TCH (mmol/L) & 0.076 & 0.021 \\
TG $(\mathrm{mmol} / \mathrm{L})$ & 0.052 & 0.110 \\
HDL-C (mmo//L) & -0.056 & 0.088 \\
LDL-C (mmol/L) & 0.086 & 0.009 \\
Apo-A (g/L) & -0.114 & 0.001 \\
Apo-B $(\mathrm{g} / \mathrm{L})$ & 0.060 & 0.068 \\
FBG $(\mathrm{mmol} / \mathrm{L})$ & 0.187 & $<0.001$ \\
miR-125b & -0.032 & 0.334 \\
miR-122 & $<0.001$ & 0.985 \\
miR-214 & -0.056 & 0.089 \\
miR-133b & 0.002 & 0.940 \\
miR-221 & -0.106 & 0.001 \\
miR-21 & -0.011 & 0.746 \\
miR-155 & -0.032 & 0.325 \\
miR-106a & -0.060 & 0.066 \\
miR-130a & -0.073 & 0.026 \\
\hline
\end{tabular}

biochemical characteristics of patients according to Gensini Score; quartile values were used as cut-off points. The results suggest that distributions of age $(p=<0.001)$, CK-MB ( $p=$ $<0.001)$, LDL-c $(p=0.036)$, apolipoprotein A ( $p=0.005)$, fasting glucose $(p=<0.001)$, miR$221(p=0.002)$, and miR-130a $(p=0.042)$ were significantly different among the various groups, and the gender distribution $(p=0.002)$ was significantly different among the groups. In addition, no difference was found regarding the distributions of TCH ( $p=0.163)$, TG ( $p=$ $0.126)$, HDL-C ( $p=0.176)$, apolipoprotein B $(p=0.474)$, miR-125b $(p=0.156), \operatorname{miR}-122(p=$ $0.188), \operatorname{miR}-214(p=0.283), \operatorname{miR}-133 b(p=0.192), \operatorname{miR}-21(p=0.963), \operatorname{miR}-155(p=0.213)$, and miR-106a among the various groups.

\section{Correlations of Gensini Score with clinical characteristics and circulating miRNAs}

As shown in Table 3, results of the Spearman correlation analyses indicated that Gensini Score was positively associated with age $(r=0.152, p=<0.001)$, CK-MB $(r=0.144$, $p=0.002)$, TCH ( $r=0.076, p=0.021)$, LDL-C $(r=0.086, p=0.009)$, and FBG $(r=0.187, p$ $=<0.001)$. Gensini Score was negatively associated with apolipoprotein A $(r=-0.092, p=$ 0.005), miR-221 ( $r=-0.106, p=0.001)$, and miR-130a $(r=-0.073, p=0.026)$. 
Table 4. Correlations between miRNAs and features of the study population: results of Spearman analyses. CK-MB, MB isoenzyme of creatine kinase; TCH, total cholesterol; TG, triglyceride; HDL-C, fasting high-density lipoprotein cholesterol; LDL-C, fasting low-density lipoprotein cholesterol; FBG, fasting blood glucose. The value of each miRNA means the relative amount calculated by $2^{-\Delta c t}$ method

\begin{tabular}{|c|c|c|c|c|c|c|c|c|c|c|c|c|c|c|c|c|c|c|}
\hline \multirow{2}{*}{ miRNAs } & \multicolumn{2}{|c|}{ Age (years) } & \multicolumn{2}{|c|}{ CK-MB (U/L) } & \multicolumn{2}{|c|}{$\mathrm{TCH}(\mathrm{mmol} / \mathrm{L})$} & \multicolumn{2}{|c|}{$\mathrm{TG}(\mathrm{mmol} / \mathrm{L})$} & \multicolumn{2}{|c|}{$\mathrm{HDL}-\mathrm{C}(\mathrm{mmol} / \mathrm{L})$} & \multicolumn{2}{|c|}{ LDL-C (mmol/L) } & \multicolumn{2}{|c|}{ Аро-A (g/L) } & \multicolumn{2}{|c|}{ Аро-B (g/L) } & \multicolumn{2}{|c|}{ FBG $(\mathrm{mmol} / \mathrm{L})$} \\
\hline & $R$ & $P$ & $R$ & $P$ & $R$ & $P$ & $R$ & $P$ & $R$ & P & $R$ & $P$ & $R$ & $P$ & $R$ & $P$ & $R$ & $P$ \\
\hline miR-125b & -0.033 & 0.314 & 0.062 & 0.060 & -0.065 & 0.046 & -0.031 & 0.341 & -0.024 & 0.456 & -0.057 & 0.084 & 0.001 & 0.968 & 0.018 & 0.575 & 0.009 & 0.791 \\
\hline miR-122 & 0.045 & 0.167 & 0.051 & 0.122 & 0.043 & 0.193 & 0.041 & 0.212 & 0.099 & 0.002 & 0.040 & 0.225 & $<0.001$ & 0.987 & 0.074 & 0.024 & 0.024 & 0.457 \\
\hline miR-214 & 0.012 & 0.712 & 0.020 & 0.544 & -0.047 & 0.149 & -0.077 & 0.019 & -0.017 & 0.607 & -0.039 & 0.238 & 0.049 & 0.138 & 0.064 & 0.050 & 0.024 & 0.462 \\
\hline miR-133b & 0.067 & 0.042 & 0.051 & 0.122 & -0.026 & 0.422 & $<0.001$ & 0.989 & 0.082 & 0.012 & 0.015 & 0.651 & 0.090 & 0.006 & 0.094 & 0.004 & 0.024 & 0.462 \\
\hline miR-221 & -0.045 & 0.172 & 0.037 & 0.261 & -0.052 & 0.110 & -0.018 & 0.576 & -0.063 & 0.055 & -0.082 & 0.012 & 0.018 & 0.576 & 0.081 & 0.013 & 0.022 & 0.507 \\
\hline miR-21 & -0.052 & 0.112 & -0.058 & 0.078 & -0.013 & 0.702 & 0.018 & 0.589 & -0.007 & 0.835 & -0.017 & 0.603 & -0.023 & 0.492 & 0.117 & $<0.001$ & 0.006 & 0.846 \\
\hline miR-155 & -0.035 & 0.291 & -0.006 & 0.044 & -0.014 & 0.680 & 0.086 & 0.008 & 0.020 & 0.545 & -0.034 & 0.304 & -0.025 & 0.446 & 0.104 & 0.001 & -0.004 & 0.896 \\
\hline & -0.081 & 0.014 & -0.060 & 0.065 & -0.032 & 0.325 & 0.004 & 0.893 & -0.058 & 0.078 & -0.057 & 0.084 & 0.038 & 0.245 & 0.044 & 0.175 & 0.049 & 0.134 \\
\hline miR-130a & -0.059 & 0.073 & -0.005 & 0.888 & -0.018 & 0.575 & -0.006 & 0.853 & -0.034 & 0.293 & -0.023 & 0.477 & 0.054 & 0.102 & 0.063 & 0.055 & 0.059 & 0.070 \\
\hline
\end{tabular}

Table 5. Correlations between miRNAs and miRNAs: results of the Spearman analyses. The value of each miRNA means the relative amount calculated by $2^{-\Delta c t}$ method

\begin{tabular}{|c|c|c|c|c|c|c|c|c|c|c|c|c|c|c|c|c|c|c|}
\hline \multirow{2}{*}{ miRNAs } & \multicolumn{2}{|c|}{ miR-125b } & \multicolumn{2}{|c|}{ miR-122 } & \multicolumn{2}{|c|}{ miR-214 } & \multicolumn{2}{|c|}{ miR-133b } & \multicolumn{2}{|c|}{ miR-221 } & \multicolumn{2}{|c|}{ miR-21 } & \multicolumn{2}{|c|}{ miR-155 } & \multicolumn{2}{|c|}{ miR-106a } & \multicolumn{2}{|c|}{ miR-130a } \\
\hline & $R$ & $P$ & $R$ & $P$ & $R$ & $P$ & $R$ & $P$ & $R$ & $P$ & $R$ & $P$ & $R$ & $P$ & $R$ & $P$ & $R$ & $P$ \\
\hline niR-125b & 1 & & 0.281 & $<0.001$ & 0.534 & $<0.001$ & .440 & $<0.001$ & 0.377 & $<0.001$ & 0.036 & 0.279 & 0.070 & 0.320 & .206 & $<0.001$ & 0.281 & $<0.001$ \\
\hline & & & 1 & & & & & & & & & & & & & & & \\
\hline & & & & & 1 & & & & & & & & & & & & & \\
\hline & & & & & & & 1 & & & & & & & & & & & \\
\hline & & & & & & & & & & & & & & & & & & \\
\hline & & & & & & & & & 0 & & 1 & - & 0.617 & $<0.001$ & 0.618 & & .486 & $<0.001$ \\
\hline & & & & & & & & & & & & & & & 0.510 & $<0.001$ & & \\
\hline & & & & & & & & & & & & & & & 1 & - & 0.715 & $<0.001$ \\
\hline miR-130a & 0.281 & $<0.001$ & 0.059 & 0.073 & 0.322 & $<0.001$ & 0.247 & $<0.001$ & 0.695 & $<0.001$ & 0.486 & $<0.001$ & 0.430 & $<0.001$ & 0.715 & $<0.001$ & 1 & - \\
\hline
\end{tabular}

Table 6. Receiver operating characteristic curve analyses for the predicting of CHD prevalence. $C I$, confidence interval; $A U C$, area under the receiver operating characteristic curve; CHD, coronary heart disease; CK-MB, MB isoenzyme of creatine kinase; TCH, total cholesterol; TG, triglyceride; HDL-C, fasting high-density lipoprotein cholesterol; LDL-C, fasting low-density lipoprotein cholesterol; Apo-A, apolipoprotein A; Apo-B, apolipoprotein A; FBG, fasting blood glucose. The value of each miRNA means the relative amount calculated by $2^{-\Delta c t}$ method. $A U C$, the closer it is to 0.5 , the less predictive it is

\begin{tabular}{lcc}
\hline Variables & AUC $(95 \% \mathrm{Cl})$ & $P$ value \\
\hline Age (years) & $0.587(0.547-0.626)$ & $<0.001$ \\
CK-MB (U/L) & $0.560(0.522-0.599)$ & 0.003 \\
TCH (mmol/L) & $0.523(0.483-0.562)$ & 0.268 \\
TG (mmol/L) & $0.530(0.489-0.571)$ & 0.145 \\
HDL-C (mmol/L) & $0.555(0.515-0.594)$ & 0.008 \\
LDL-C (mmol/L) & $0.534(0.494-0.574)$ & 0.095 \\
Apo-A (g/L) & $0.534(0.503-0.583)$ & 0.036 \\
Apo-B (g/L) & $0.525(0.486-0.564)$ & 0.224 \\
FBG (mmol/L) & $0.612(0.574-0.649)$ & $<0.001$ \\
miR-125b & $0.510(0.470-0.550)$ & 0.633 \\
miR-122 & $0.527(0.488-0.566)$ & 0.186 \\
miR-214 & $0.519(0.478-0.559)$ & 0.363 \\
miR-133b & $0.513(0.474-0.553)$ & 0.517 \\
miR-221 & $0.569(0.529-0.609)$ & 0.001 \\
miR-21 & $0.502(0.463-0.542)$ & 0.909 \\
miR-155 & $0.504(0.501-0.579)$ & 0.049 \\
miR-106a & $0.533(0.494-0.573)$ & 0.105 \\
miR-130a & $0.566(0.525-0.606)$ & 0.001 \\
\hline
\end{tabular}

Spearman correlations of miRNAs with clinical characteristics and the other miRNAs

As shown in Table 4 and 5, using nonparametric Spearman correlation tests for all collected variables, we found that in all subjects, miR-221 was associated with LDL-C $(r=$ $-0.082, p=0.012)$, apolipoprotein B ( $r=0.081, p=0.013)$, miR-125b $(r=0.377, p=<0.001)$, miR-122 ( $r=0.266, p=<0.001)$, miR-214 ( $r=0.416, p=<0.001)$, miR-133b $(r=0.338, p$ $=<0.001), \operatorname{miR}-21(r=0.390, p=<0.001), \operatorname{miR}-155(r=0.319, p=<0.001), \operatorname{miR}-106 \mathrm{a}(r=$ $0.551, p=<0.001)$, miR-130a $(r=0.695, p=<0.001)$. miR-155 was associated with CK-MB $(r$ $=-0.006, p=0.044)$, TG $(r=0.086, p=0.008)$, apolipoprotein $\mathrm{B}(r=0.104, p=0.001)$, miR125b $(r=-0.070, p=0.032), \operatorname{miR}-155(r=0.069, p=0.036), \operatorname{miR}-214(r=-0.131, p=<0.001)$, miR-221 ( $r=0.319, p=<0.001), \operatorname{miR}-21(r=0.617, p=<0.001)$, miR-106a $(r=0.510, p=$ $<0.001)$, miR-130a $(r=0.430, p=<0.001)$; miR-130a were associated with miR-125b $(r=$ $0.281, p=<0.001)$, miR-214 ( $r=0.322, p=<0.001)$, miR-133b $(r=0.247, p=<0.001)$, miR$221(r=0.695, p=<0.001)$, miR-21 $(r=0.486, p=<0.001)$, miR-155 $(r=0.430, p=<0.001)$, $\operatorname{miR}-106 \mathrm{a}(r=0.715, p=<0.001)$.

Predictors of CHD prevalence: results of ROC analyses

To further explore the applicability of circulating miRNAs and classical risk factors as potential diagnostic biomarkers of CHD, subsequent ROC analyses were performed, and the 


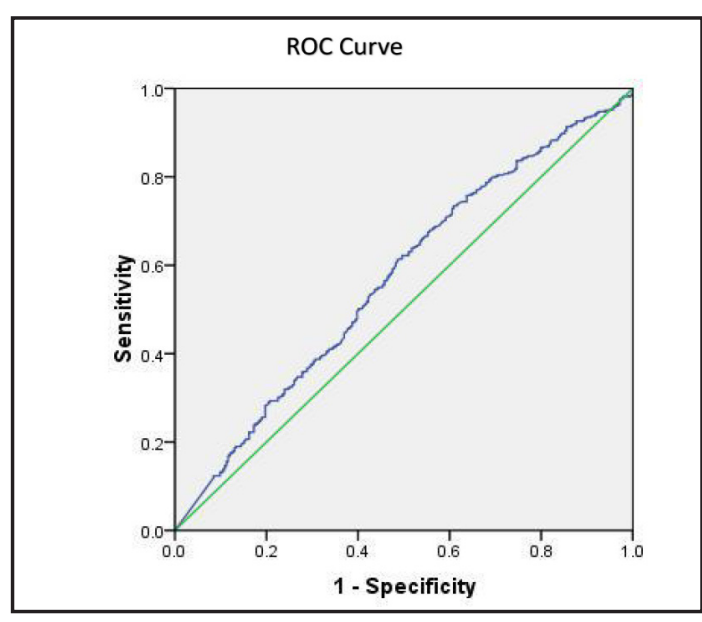

Fig. 1. The receiver operating characteristic curve for the predictive efficacy of miR-221 for CHD prevalence.

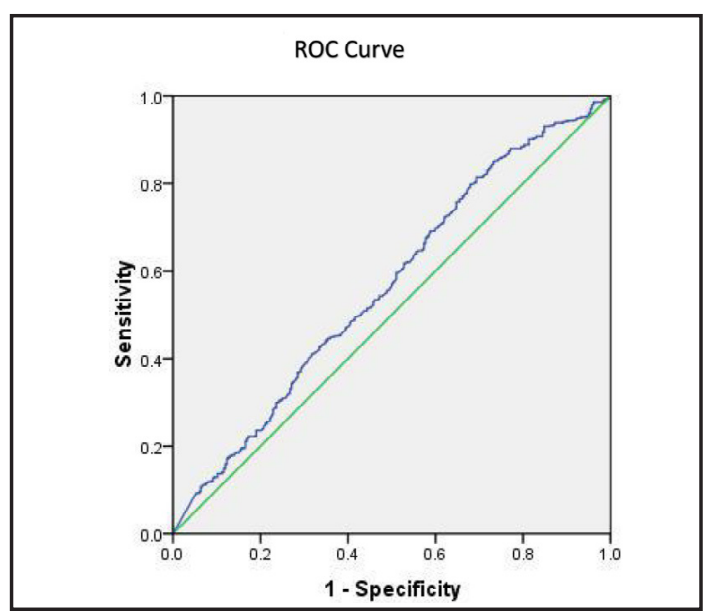

Fig. 3. The receiver operating characteristic curve for the predictive efficacy of miR-221 for CHD prevalence.

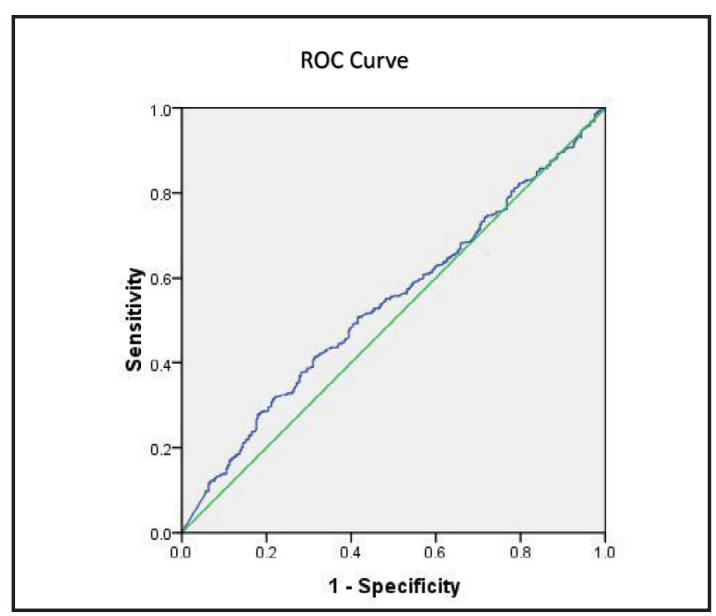

Fig. 2. The receiver operating characteristic curve for the predictive efficacy of miR-221 for CHD prevalence.

Table 7. The optimal cut-off and the Youden index. CK-MB, MB isoenzyme of creatine kinase; HDL-C, fasting high-density lipoprotein cholesterol; Apo-A, apolipoprotein A; FBG, fasting blood glucose. The value of each miRNA means the relative amount calculated by $2^{-\Delta c t}$ method

\begin{tabular}{lcccc}
\hline Variables & Cutoff & Sensitivity & Specificity & Youdenindex \\
\hline Age (years) & 59.5 & 0.574 & 0.556 & 0.130 \\
CK-MB (U/L) & 25.9 & 0.202 & 0.940 & 0.142 \\
HDL-C (mmol/L) & 1.255 & 0.697 & 0.418 & 0.115 \\
Apo-A (g/L) & 1.155 & 0.778 & 0.287 & 0.065 \\
FBG (mmol/L) & 5.445 & 0.423 & 0.799 & 0.222 \\
miR-155 & 100.427 & 0.687 & 0.414 & 0.101 \\
miR-221 & 0.114 & 0.514 & 0.613 & 0.127 \\
miR-130a & 6.521 & 0.306 & 0.815 & 0.121 \\
\hline
\end{tabular}

results are shown in Table 6. The AUC for the predicting of CHD prevalence was 0.587 for age (95 \% CI: 0.547-0.626, $p=<0.001$ ); 0.560 for CK-MB (95 \% CI: 0.522-0.599, $p=0.003$ ); 0.555 for HDL-C (95 \% CI: 0.515-0.594, $p=0.008$ ); 0.534 for Apo-A (95 \% CI: 0.503-0.583, $p=0.036$ ); and 0.612 for FBG (95\% CI: 0.574-0.649, $p=<0.001)$. Of all the miRNAs, miR221 had an $A U C$ of 0.569 (95 \% CI: 0.529-0.609, $p=0.001$ ) (Fig. 1), miR-155 had an AUC of0.504 (95 \% CI: 0.501-0.579, $p=0.049$ ) (Fig. 2) and miR-130a had an AUC of 0.566 (95\% CI: $0.525 \sim 0.606, p=0.001$ ) (Fig. 3 ). The optimal cut-off value, the sensitivity, the specificity and Youden index of age, CK-MB, HDL-C, Apo-A, FBG, miR-221, miR-155 and miR-130a are shown in Table 7.

\section{Predictors of CHD prevalence: results of logistic regression analyses}

We performed a miRNAs score analysis to evaluate the associations between the combination of the plasma miRNAs and CHD. Briefly, the miRNAs score of each miRNA (miR221, miR-155 and miR-130a), was set to 1 if its concentration was lower than the optimal cutoff value for the corresponding miRNA. Otherwise the score was set to 0 . Thus, the miRNAs score was the sum of the score of miR-221, miR-155 and miR-130a. The characteristics of the study population categorized by the miRNAs score were shown in Table 8.

\section{KARGER}


Table 8. Characteristics of the study population categorized by the miRNAs score: results of K independent sample test. CK-MB, MB isoenzyme of creatine kinase; TCH, total cholesterol; TG, triglyceride; HDL-C, fasting high-density lipoprotein cholesterol; LDL-C, fasting low-density lipoprotein cholesterol; Apo-A, apolipoprotein A; Apo-B, apolipoprotein A; FBG, fasting blood glucose

\begin{tabular}{|c|c|c|c|c|c|c|}
\hline \multirow{2}{*}{ Characteristics } & \multicolumn{4}{|c|}{ miRNAs score } & \multirow{2}{*}{ Statistical parameter } & \multirow{2}{*}{$P$ value } \\
\hline & $0(n=150)$ & $1(\mathrm{n}=194)$ & $2(\mathrm{n}=341)$ & $3(\mathrm{n}=247)$ & & \\
\hline Age (years) & $59.50(52.00-67.00)$ & $60.00(52.00-68.00)$ & $61.00(51.00-69.00)$ & $61.00(53.00-70.00)$ & 4.045 & 0.257 \\
\hline Gender $(\mathrm{M} / \mathrm{F})$ & $112 / 38$ & $141 / 53$ & $256 / 85$ & $172 / 75$ & 2.385 & 0.496 \\
\hline CK-MB (U/L) & $16.50(13.00-21.70)$ & $17.00(13.68-21.03)$ & $16.00(13.00-21.00)$ & $17.00(13.00-22.00)$ & 3.254 & 0.354 \\
\hline $\mathrm{TCH}(\mathrm{mmol} / \mathrm{L})$ & $4.51(3.68-5.37)$ & $4.74(4.07-5.45)$ & $4.63(3.84-5.43)$ & $4.72(3.94-5.67)$ & 5.253 & 0.154 \\
\hline $\mathrm{TG}(\mathrm{mmol} / \mathrm{L})$ & $1.73(1.24-2.61)$ & $1.67(1.16-2.47)$ & $1.84(1.28-2.48)$ & $1.75(1.17-2.42)$ & 3.950 & 0.267 \\
\hline HDL-C (mmol/L) & $1.29(1.10-1.57)$ & $1.36(1.14-1.64)$ & $1.33(1.10-1.62)$ & $1.45(1.18-1.69)$ & 9.549 & 0.023 \\
\hline LDL-C (mmol/L) & $2.63(2.07-3.39)$ & $2.75(2.27-3.34)$ & $2.73(2.15-3.32)$ & $2.95(2.15-3.69)$ & 6.775 & 0.079 \\
\hline Apo-A(g/L) & $1.29(1.14-1.46)$ & $1.29(1.17-1.46)$ & $1.28(1.13-1.43)$ & $1.28(1.14-1.48)$ & 2.023 & 0.568 \\
\hline Apo-B(g/L) & $0.86(0.74-1.01)$ & $0.97(0.83-1.09)$ & $0.90(0.75-1.06)$ & $0.85(0.73-1.03)$ & 22.115 & $<0.001$ \\
\hline $\mathrm{FBG}(\mathrm{mmol} / \mathrm{L})$ & $5.06(4.71-5.93)$ & $5.00(4.58-5.84)$ & $5.10(4.62-5.99)$ & $5.08(4.50-5.94)$ & 1.184 & 0.757 \\
\hline Gensini score & $7.50(0.00-36.00)$ & $12.00(1.50-36.25)$ & $19.00(4.00-50.50)$ & $5.08(4.50-5.94)$ & 19.016 & $<0.001$ \\
\hline
\end{tabular}

Table 9. Univariatelogistic regression analysis for the predictors of CHD prevalence. $\mathrm{CHD}$, coronary heart disease; CK-MB, MB isoenzyme of creatine kinase; TCH, total cholesterol; TG, triglyceride; HDL-C, fasting high-density lipoprotein cholesterol; LDL-C, fasting lowdensity lipoprotein cholesterol; Apo-A, apolipoprotein A; Apo-B, apolipoprotein A; FBG, fasting blood glucose. The value of each miRNA means the relative amount calculated by $2^{-\Delta c t}$ method

\begin{tabular}{|c|c|c|c|c|}
\hline Characteristics & $\beta$-coefficient & $O R$ & $95 \% C I$ & $P$ value \\
\hline Age (years) $(<59.5$ v.s. $\geq 59.5)$ & 0.525 & 1.690 & $1.276,2.239$ & $<0.001$ \\
\hline Gender(F/M) & 0.606 & 1.832 & $1.352,2.483$ & $<0.001$ \\
\hline CK-MB (U/L) $(<25.9$ v.s. $\geq 25.9)$ & 1.381 & 3.980 & $2.351,6.737$ & $<0.001$ \\
\hline TCH $(\mathrm{mmol} / \mathrm{L})(<4.63 \mathrm{vs} \geq 4.63)$ & 0.120 & 1.127 & $0.853,1.490$ & 0.400 \\
\hline $\mathrm{TG}(\mathrm{mmol} / \mathrm{L})(<1.76 \mathrm{vs} \geq 1.76)$ & 0.203 & 1.225 & $0.926,1.620$ & 0.155 \\
\hline HDL-C $(\mathrm{mmol} / \mathrm{L})(>1.255 \mathrm{vs} \leq 1255)$ & 0.504 & 1.655 & $1.229,2.228$ & 0.001 \\
\hline LDL-C $(\mathrm{mmol} / \mathrm{L})(<2.75 \mathrm{vs} \geq 2.75)$ & 0.168 & 1.183 & $0.895,1.565$ & 0.237 \\
\hline Apo-A $(g / L)(>1.155$ vs $\leq 1.155)$ & 0.345 & 1.412 & $1.018,1.960$ & 0.039 \\
\hline Apo-B $(\mathrm{g} / \mathrm{L})(<0.90 \mathrm{vs} \geq 0.90)$ & 0.084 & 1.087 & $0.823,1.437$ & 0.556 \\
\hline $\mathrm{FBG}(\mathrm{mmol} / \mathrm{L})(<5.445 \mathrm{vs} \geq 5.445)$ & 1.071 & 2.918 & $2.098,4.057$ & $<0.001$ \\
\hline miR-125b $(>0.034$ v.s. $\leq 0.0 .034)$ & 0.026 & 1.027 & $0.777,1.357$ & 0.853 \\
\hline miR-122 (>0.191 v.s. $\leq 0.191)$ & 0.162 & 1.176 & $0.889,1.555$ & 0.255 \\
\hline miR-214 (>0.003 v.s. $\leq 0.003)$ & 0.130 & 1.138 & $0.861,1.505$ & 0.363 \\
\hline miR-133b $(>0.167$ v.s. $\leq 0.167)$ & 0.217 & 1.242 & $0.939,1.643$ & 0.128 \\
\hline miR-221 (>0.114 v.s. $\leq 0.114)$ & 0.508 & 1.663 & $1.255,2.202$ & $<0.001$ \\
\hline miR-21 (>1.840 v.s. $\leq 1.840)$ & -0.087 & 0.916 & $0.693,1.211$ & 0.540 \\
\hline miR-155 (> 100.427 v.s. $\leq 100.427)$ & 0.419 & 1.520 & $1.132,2.042$ & 0.005 \\
\hline miR-106a (>1.542 v.s. $\leq 1.542)$ & 0.162 & 1.176 & $0.889,1.555$ & 0.255 \\
\hline miR-130a $(>6.521$ v.s. $\leq 6.521)$ & 0.664 & 1.943 & $1.410,2.678$ & $<0.001$ \\
\hline miRNAs score & 0.326 & 1.386 & $1.208,1.589$ & $<0.001$ \\
\hline \multicolumn{5}{|l|}{0} \\
\hline 1 & 0.752 & 1.566 & $1.010,2.426$ & 0.045 \\
\hline 2 & 0.990 & 2.121 & $1.422,3.164$ & $<0.001$ \\
\hline 3 & 0.214 & 2.691 & $1.738,4.167$ & $<0.001$ \\
\hline
\end{tabular}

Table 10. Multivariate logistic regression analysis for the predictors of CHD prevalence. CHD, coronary heart disease; CK-MB, MB isoenzyme of creatine kinase; HDL-C, fasting high-density lipoprotein cholesterol;FBG, fasting blood glucose. The value of each miRNA means the relative amount calculated by $2^{-\Delta c t}$ method

\begin{tabular}{lllll}
\hline Characteristics & $\beta$-coefficient & $O R$ & $95 \%$ CI & $P$ value \\
\hline Age (years) $(<59.5$ v.s. $\geq 59.5)$ & 0.650 & 1.916 & $1.411,2.601$ & $<0.001$ \\
Gender(F/M) & 0.652 & 1.918 & $1.375,2.676$ & $<0.001$ \\
CK-MB (U/L) $(<25.9$ v.s. $\geq 25.9)$ & 1.317 & 3.730 & $2.150,6.472$ & $<0.001$ \\
HDL-C (mmol/L) $(>1.255$ vs $\leq 1255)$ & 0.595 & 1.814 & $1.315,2.502$ & $<0.001$ \\
FBG (mmol/L) $<5.445$ vs $\geq 5.445)$ & 0.997 & 2.711 & $1.915,3.837$ & $<0.001$ \\
miR-130a $(>6.521$ v.s. $\leq 6.521)$ & 0.878 & 2.405 & $1.691,3.421$ & $<0.001$ \\
\hline
\end{tabular}

The results of the univariate logistic regression analysis for the predictors of CHD prevalence are shown in Table 9. Older age, male, higher CK-MB, lower HDL-C, and lower Apo-A level were potential predictors of CHD. Moreover, lower circulating miR-221(OR, 1.663; $95 \% C I, 1.255-2.202, p=<0.001)$, miR-155(OR, 1.520; $95 \% C I, 1.132-2.042, p=0.005)$, and miR-130a (OR, 1.943; $95 \% C I, 1.410-2.678, p=<0.001)$ were significantly associated with $\mathrm{CHD}$ in the univariable analysis.

It was assumed that subjects with a miRNA score of 0 have a background risk of CHD $(O R$ is assumed to be 1 ); the results of the univariate logistic regression analysis indicated that the relative risk of CHD among the subjects with miRNAs scores of 1 and 2 and were 
Table 11. The relationship between miR-221, miR-155, and miR-130a. SI, synergy index for interaction in an additive model; SI, SI, synergy index for interaction in a multiplicative model; RERI, relative excess risk due to interaction; $A P$, proportion of disease attributable to interaction

\begin{tabular}{|c|c|c|c|c|c|}
\hline miR-221 & miR-155 & CHDs & Controls & OR $(95 \% C I)$ & $P$ value \\
\hline 0 & 0 & 178 & 114 & 1 & \\
\hline $\mathbf{0}$ & 1 & 74 & 32 & $1.481(0.919-2.386)$ & 0.106 \\
\hline 1 & 0 & 202 & 80 & $1.617(1.140-2.294)$ & 0.007 \\
\hline 1 & 1 & 194 & 58 & $2.142(1.471-3.120)$ & $<0.001$ \\
\hline \multicolumn{6}{|c|}{$S I=1.04 \quad S I M=0.89$ RERI $=0.04 \quad A P=0.02$} \\
\hline miR-221 & miR-130a & CHDs & Controls & OR $(95 \% C I)$ & $P$ value \\
\hline $\mathbf{0}$ & 0 & 99 & 79 & 1 & \\
\hline $\mathbf{0}$ & 1 & 153 & 67 & $1.822(1.207-2.752)$ & 0.004 \\
\hline 1 & 0 & 21 & 8 & $2.095(0.881-4.982)$ & 0.094 \\
\hline 1 & 1 & 375 & 130 & $2.302(1.611-3.288)$ & $<0.001$ \\
\hline \multicolumn{6}{|c|}{$S I=0.68 \quad S I M=0.60 \quad R E R I=-0.62 \quad A P=-0.27$} \\
\hline miR-130a & miR-155 & CHDs & Controls & OR $(95 \% \mathrm{CI})$ & $P$ value \\
\hline 0 & 0 & 100 & 74 & 1 & \\
\hline $\mathbf{0}$ & 1 & 280 & 120 & $1.138(0.532-2.435)$ & 0.738 \\
\hline 1 & 0 & 20 & 13 & $1.727(1.194-2.497)$ & 0.004 \\
\hline 1 & 1 & 248 & 77 & $2.383(1.606-3.536)$ & $<0.001$ \\
\hline \multicolumn{6}{|c|}{$S I=1.60 \quad S I M=1.21 \quad R E R I=0.52 \quad A P=0.22$} \\
\hline
\end{tabular}

1.566 (OR, 1.566; $95 \% C I, 1.010-2.426, p=0.045), 2.121(O R, 2.121 ; 95 \% C I, 1.422-3.164, p$ $=<0.001)$ and $2.691(O R, 2.691 ; 95 \% C I, 1.738-4.167, p=<0.001)$.

A multivariate logistic regression analysis (Forward: Conditional method) was used to identify the risk factors for CHD among the entire population. In the multivariate model involving the entire population, we included the following variables: age, gender, TCH, TG, FBG, HDL-C, LDL-C, CK-MB, Apo-A, Apo-B, miR-122, miR-133b, miR-214, miR-21, miR-106a, miR-130a, miR-155, miR-221, and miR-125b. The results are reported as adjusted $O R s$ and 95 $\%$ confidence intervals in Table 10. Older age, male, higher CK-MB, lower HDL-C, higher FBG, and lower miR-130a (OR, 2.405; $95 \% C I, 1.691-3.421, p=<0.001)$ remained independently associated with the risk of CHD.

Interaction between miR-221, miR-155 and miR-130a

The analysis of the possible positive/negative associations between miR-221, miR155 and miR-130a are expressed in Table 11. According to the results of the $R O C$ analysis, subjects with a miR-221, miR-155 and miR-130a concentration below $0.114,100.427$, and 6.521 were consideredto have an elevated risk of CHD, respectively. Regarding the baseline risk for subjects unexposed to risk expression of miR-221, miR-155 or miR-130a (reference category, 1.0), the $O R$ estimating the joint effect of miR-221 with miR-155, miR-221 with miR-130a, and miR-155 with miR-130a was significantly higher than the ORs estimating the effects of each factor in the absence of the other respectively. A positive association between miR-130a and miR-155 was found ( $S I=1.60, S I M=1.21$ and $A P=0.22)$, and in these groups, the proportion of CHD attributable to the interaction between miR-130a and miR-155 was as high as $22 \%$. A negative interaction was found between miR-221 and miR-130a $(S I=0.68$, $S I M=0.60$ and $A P=0.27$ ).

Functional annotation of the target genes for miR-221

To further identify the putative function of the predicted target genes for miR-221, G0 analysis was performed. Eighteen different biological processes, four cellular components and six different molecular functions were predicted (Fig. 4). The most significantly enriched GO terms were involved in three main categories: protein transport, protein localization, and establishment of protein localization. These results suggested that regulation of protein transport and localization played a vital role in the process of the CHD for miR-221. In addition, KEGG analysis was further conducted to elucidate the biological pathways of the miR-221 target genes (Fig. 5). The categories of neurotrophin signaling pathway, $\mathrm{T}$ cell receptor signaling pathway, and ErbB signaling pathway were the most enriched pathways, which were all in the group of "signaling pathways". This suggested that miR-221 regulation of signaling pathway genes played crucial roles in the development of CHD. 
Fig. 4. GO analysis of the target genes for miR-221.

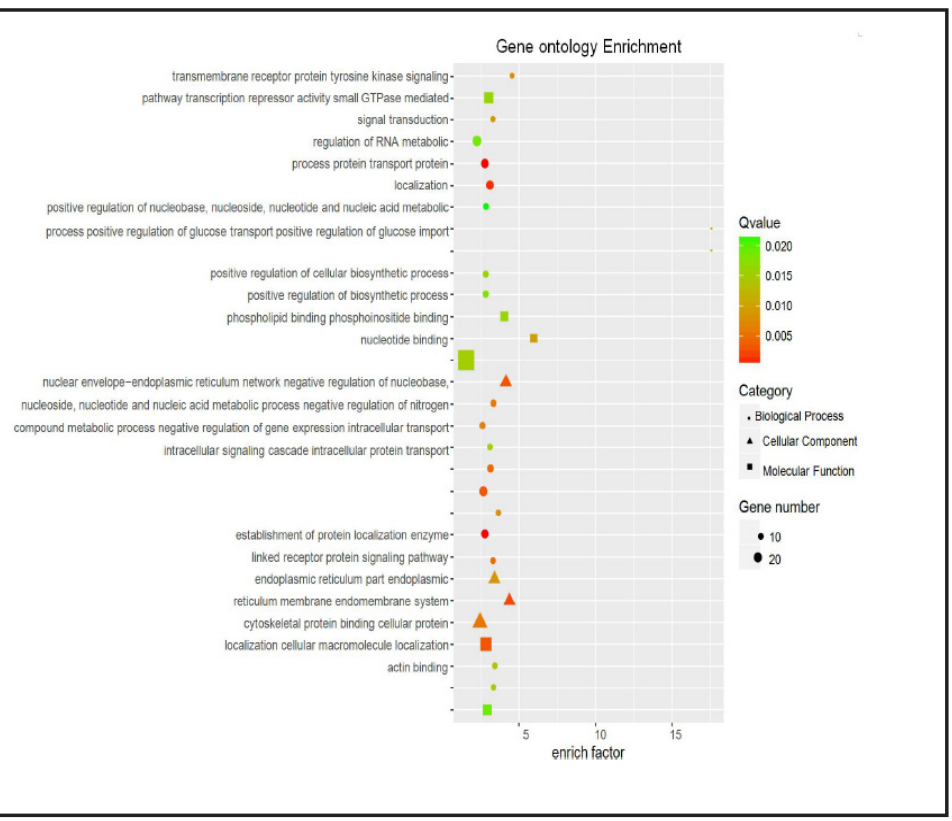

Fig. 5. KEGG pathways of the target genes for miR221.

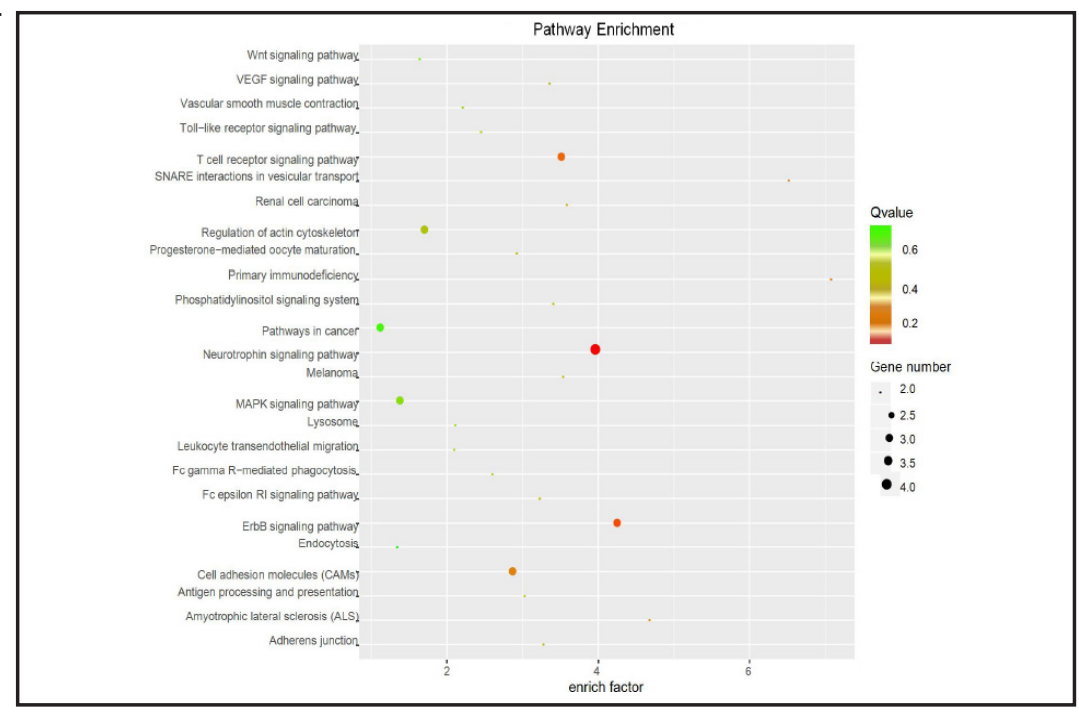

Functional annotation of the target genes for miR-155

The GO/pathway analysis results for miR-155are presented in Fig. 6 and Fig. 7. The most significantly enriched G0 terms for miR-155 involved three main categories, including enzyme binding, transmembrane receptor protein serine/threonine kinase activity, and transforming growth factor beta receptor activity. Moreover, KEGG analysis suggests that the categories of pathways in cancer, pancreatic cancer, and colorectal cancer were the most enriched pathways, which were all in the group of cancer pathways.

Functional annotation of the target genes for miR-130a

Fig. 8 indicates that the most significantly enriched GO terms for miR-130a were the three main categories of regulation of transcription, regulation of cell cycle, and regulation of protein catabolic process. These results suggest that regulation of transcription played a vital role in the process of the CHD for miR-221. In addition, KEGG analysis presented in Fig. 9 suggest that the categories of viral myocarditis, endocytosis, and neuroactive ligandreceptor interaction were the most enriched pathways. 
Fig. 6. GO analysis of the target genes for miR-155.

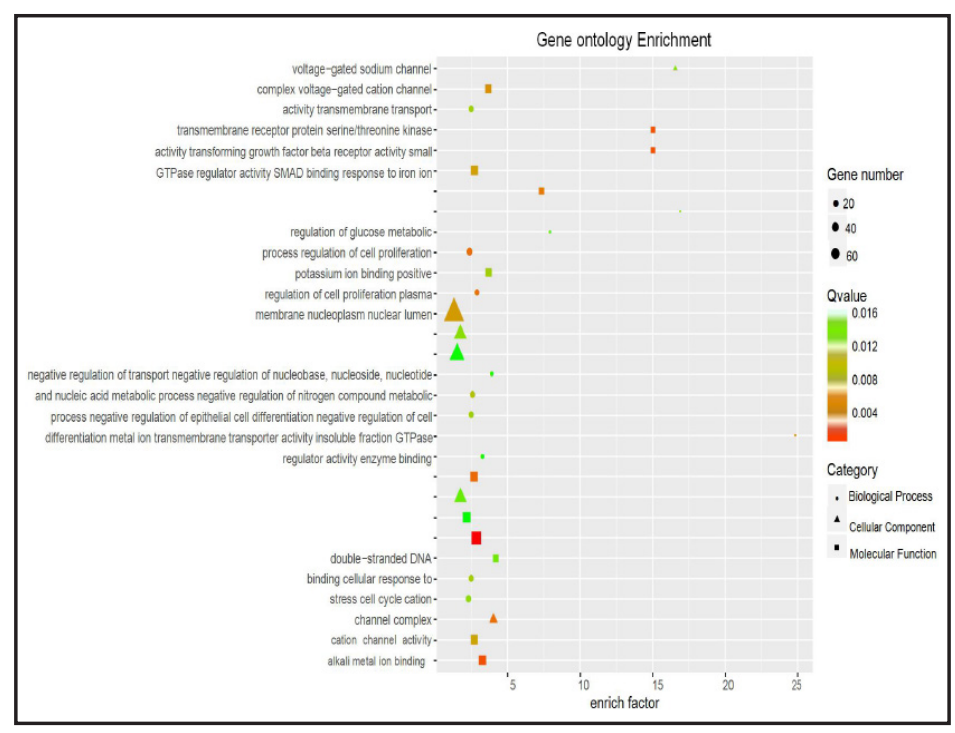

Fig. 7. KEGG pathways of the target genes for miR-155.

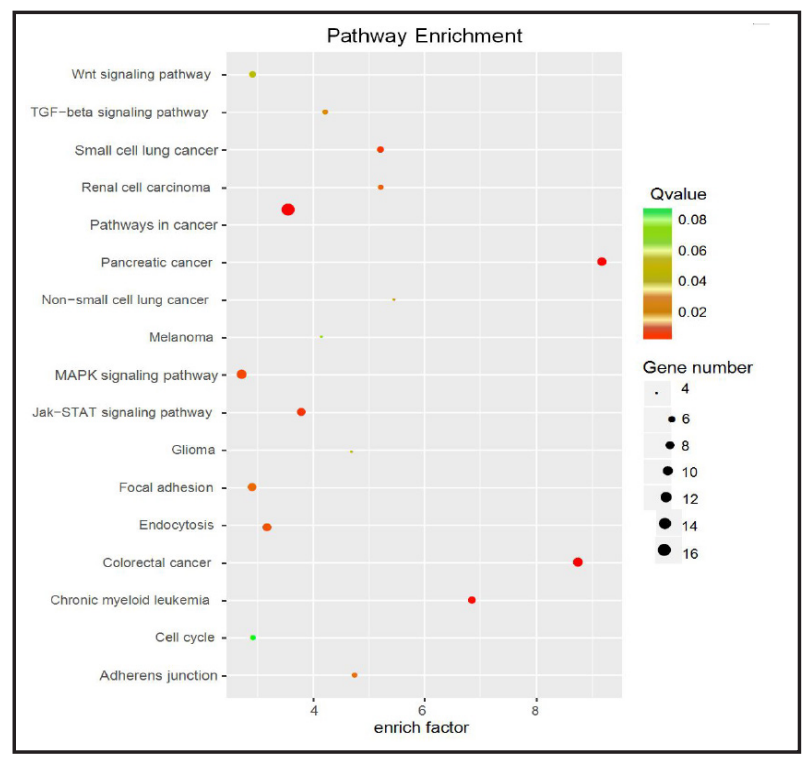

\section{Discussion}

In this study, we evaluated the predictive role of circulating miRNAs for CHD in a multiethnic population in China. We found that serum miR-155, miR-221 and miR-130a were significantly reduced in patients with CHD as compared with controls. More importantly, lower circulating miR-155, miR-221 and miR-130a were all significant predictors of CHD prevalence in this population. More importantly, miR-130a remained the independent predictor for CHD prevalence after adjustment for conventional confounding factors. To the best of our knowledge, ours is the first study that evaluates circulating miRNAs in a multiethnic population of documented CHD patients and control subjects. These results suggest that miRNAs, particularly miR-130a, may be novel biomarkers for CHD risk in this multiethnic population. Cohort studies are needed to confirm our results, and mechanism analyses are needed to determine the role of miRNAs in the pathogenesis of CHD.

miRNAs are endogenous, non-coding, and small (18-22 nucleotides) RNA molecules. miRNAs are recruited to the RNA-induced silencing complex (RISC) and regulate the output 
Fig. 8. GO analysis of the target genes for miR-130a.

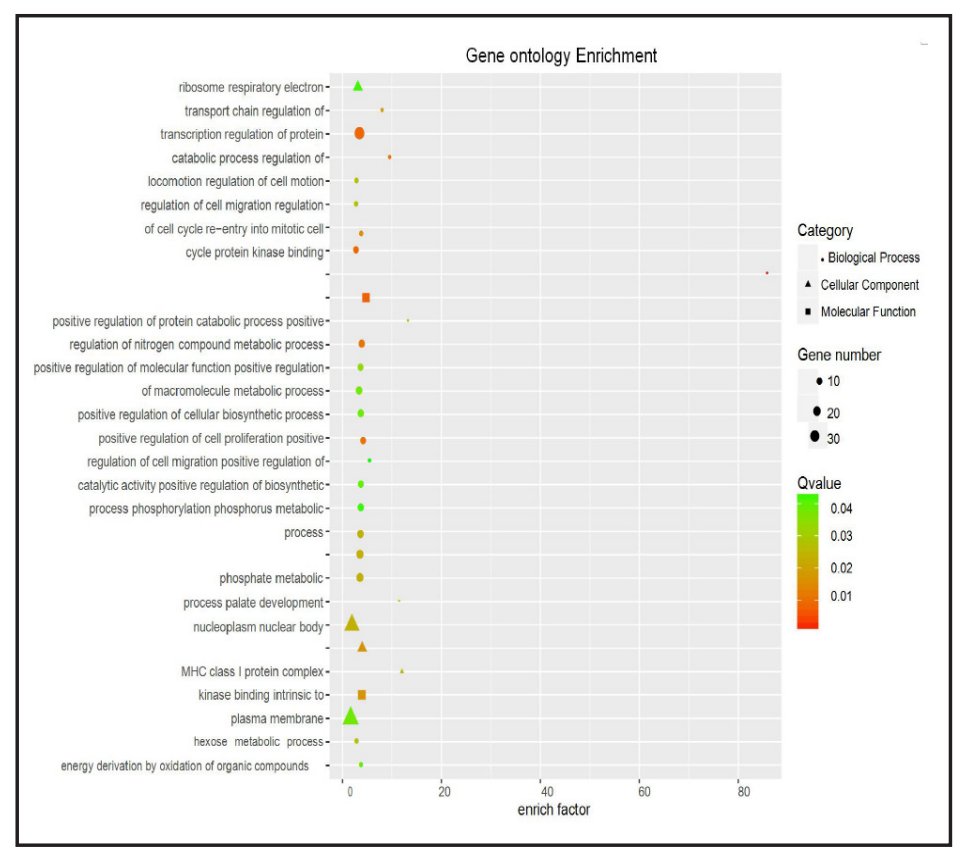

Fig. 9. KEGG pathways of the target genes for miR-130a.

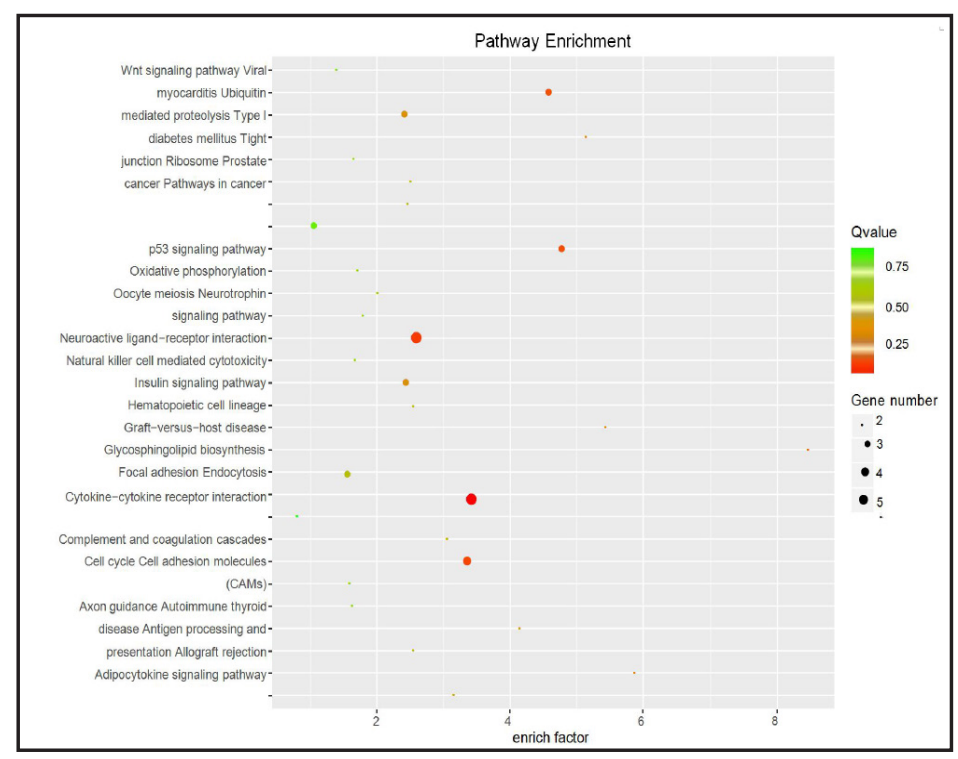

of protein-coding genes through diverse mechanisms [46]. The interaction of miRNAs with the $3^{\prime}$ untranslated region ( $3^{\prime} U T R$ ) of protein-coding genes is considered as the main mechanism of action, which usually leads to a decrease in protein output either by mRNA degradation or by translational repression [47]. Recent studies have shown that miRNAs are key regulators of gene expression. The expression levels of miRNAs have been shown to be associated with CVD [48], cancer [49], and other diseases [50]. In addition, miRNAs have been used as potential biomarkers for both non-communicable and communicable diseases [51,52]. However, to date, miRNA expression differences have not been reported in a multi-ethnic population. In the present study, we systematically explored nine CHD-related miRNAs in plasma from 932 CHD patients and non-CHD controls, with the results suggesting that the expression level of the miRNAs was not different among the various ethnic groups.

Results from our study indicate that miRNAs, particularly miR-155, miR-221 and miR130a may be novel markers of CHD. As a specific miRNA identified in human umbilical vein endothelial cells (HUVECs), miR-221 participates in the regulation of angiogenesis KARGER 
[53]. Previous findings demonstrate that miR-221 inhibits the proliferation of endothelial progenitor cells (EPC) via interactions with the MEK/ERK pathway, thereby indicating a potential role of miR-221 for maintaining the integrity of the endothelium [54]. Interestingly, treatment with atorvastatin increased EPC numbers and decreased miR-221/222 levels in patients with CHD [55]. As for miR-155, it has been demonstrated thatmiR-155 exerts an antiangiogenic but proarteriogenic function in the regulation of neovascularization via the suppression of divergent cell-specific target genes in mice [56]. In fact, a previous study has concluded that circulating miR-155 is lower in CHD patients, and inversely associated with the extent of coronary stenosis as indicated by the Gensini Scores [57]. Also, miR-130a has also been implicated in the angiogenic process $[58,59]$, demonstrating that the overexpression of miR-130a may contribute importantly to gap junction remodeling and to the pathogenesis of atrial and ventricular arrhythmias [60]. Moreover, miR-130a has been implicated in the process of cardiac development, perhaps via regulation of FOG-2 (also known as zfpm2)[61]. Results from our study further confirmed the potential association between the expression of the above three miRNAs and CHD. Moreover, expression of these miRNAs also correlated with the severity of coronary lesions as indicated by Gensini Scores. These results should be verified in future cohort studies, and the exact mechanisms underlying the role of the above miRNAs in the pathogenesis of CHD deserves further investigation.

To comprehensively investigate miR-221, miR-155, miR-130aand their targets and provide some information for further understanding the miRNA-mediated regulation network in the development of CHD, GO/pathwayenrichmentanalysis was conducted in this study. Our analysis showed that miR-221, miR-155, and miR-130a contribute to various biological processes, including the regulation of protein transport and localization, cancer related pathways, endocytosis, and neuroactive ligand-receptor interactions. Taken together, these results represented partial information on the biological function of miRNA in the occurrence and development of CHD. However, the exact mechanisms underlying the association of miRNA and CHD require further study.

Our study has limitations which should be considered when interpreting the results. Firstly, our study is cross-sectional in design. Although we adjustedfor conventional risk factors of CHD, the potential association between the aforementioned miRNAs and CHD prevalence may be confounded by residue factors. Moreover, our study only provides evidence for the potential association between the miRNAs and CHD; whether changes in the miRNAs identified are causative to the pathogenesis of CHD requires further evaluation. Finally, the results may be different according to the different populations included. Therefore, further studies are needed to confirm our results.

In summary, expression levels of miR-221, miR-155, and miR-130a were decreased in CHD patients compared with controls and may be predictive of CHD risk in this population. Our findings suggested that miR-221, miR-155, and miR-130a might play an important role in the development of CHD, serving as a potential biomarker for predicting CHD. Cohort studies are needed to confirm our results, and mechanism analyses are needed to determine the role of miRNAs in the pathogenesis of CHD.

\section{Acknowledgement}

This study received support from the National Natural Science Foundations of China (No. 81170180, 30400173 and 30971257) and the Priority Academic Program Development of Jiangsu Higher Education Institutions. Dr. En-Zhi Jia is an Assistant Fellow at the Collaborative Innovation Center for Cardiovascular Disease Translational Medicine.

\section{Disclosure Statement}

There was not any conflict of interest existing in this manuscript.

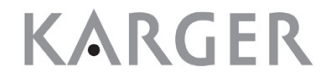




\section{Cellular Physiology Cell Physiol Biochem 2017;42:808-823 \begin{tabular}{ll|l} 
and Biochemistry Published onlıne: June 19, 2017 & $\begin{array}{l}\text { C) 2017 The Author(s). Published by S. Karger AG, Basel } \\
\text { www.karger.com/cpb }\end{array}$ \\
\hline
\end{tabular} \\ Jia et al.: Potential Biomarkers for Detection of $\mathrm{CHD}$}

\section{References}

1 Nishiguchi T, Imanishi T, Akasaka T: MicroRNAs and cardiovascular diseases.Biomed Res Int 2015;2015:682857.

2 WHO:Global Status Report on noncommunicable diseases 2014 (2015). Available at: http://www. thehealthwell.info/node/831799 (Accessed: 18th December 2015).

3 WHO:Cardiovascular diseases mortality: age-standardized death rate per 100,000 population,2000-2012(2014). Available at: http://gamapserver.who.int/gho/interactive_charts/ncd/ mortality/cvd/atlas.html (Accessed: 18th December 2015).

$\checkmark 4$ Izawa H,Amano T: Plasma microRNA-100 as a biomarker of coronary plaque vulnerability - a new generation of biomarker for developing acute coronary syndrome.Circ J2015;79:303-304.

-5 Hu YW,Hu YR,Zhao JY,Li SF,Ma X,Wu SG,Lu JB,Qiu YR,Sha YH,Wang YC,Gao JJ,Zheng L,Wang Q: An agomir of miR-144-3p accelerates plaque formation through impairing reverse cholesterol transport and promoting pro-inflammatory cytokine production.PLoS One2014;9:e94997.

-6 Van Rooij E, Sutherland LB, Thatcher JE, DiMaio JM, Naseem RH, Marshall WS, Hill JA, Olson EN:Dysregulation of microRNAs after myocardial infarction reveals a role of miR-29 in cardiac fibrosis.Proc Natl Acad Sci U S A2008;105:13027-31302.

7 Corsten MF, Dennert R, Jochems S, Kuznetsova T, Devaux Y, Hofstra L, WagnerDR, Staessen JA, Heymans S, Schroen B: Circulating microRNA-208b and microRNA-499 reflect myocardial damage incardiovascular disease.Circ Cardiovasc Genet 2010;3:499-506.

$>8$ Wei T, Folkersen L, Ehrenborg E, Gabrielsen A.MicroRNA 486-3P as a stability marker in acute coronary syndrome.Biosci Rep2016;36. pii: e00351.

-9 Creemers EE, Tijsen AJ, Pinto YM:Circulating microRNAs: novel biomarkers and extracellular communicators incardiovascular disease?Circ Res 2012;110:483-495.

10 Liu W, Ling S, Sun W, Liu T, Li Y, Zhong G, Zhao D, Zhang P, Song J, Jin X, Xu Z, Song H, Li Q, Liu S, Chai M, Dai Q, He Y, Fan Z, Zhou YJ, Li Y: Circulating microRNAs correlated with the level of coronary artery calcification in symptomatic patients. Sci Rep 2015;5:16099.

11 Liao C, Gui Y, Guo Y, Xu D: The regulatory function of microRNA-1 in arrhythmias. Mol Biosyst 2016;12:328-333.

12 Carè A, Catalucci D, Felicetti F, Bonci D, Addario A, Gallo P, Bang ML, Segnalini P, Gu Y, Dalton ND, Elia L, Latronico MV, Høydal M, Autore C, Russo MA, Dorn GW 2nd, Ellingsen O, Ruiz-Lozano P, Peterson KL, Croce CM, Peschle C, Condorelli G: MicroRNA-133 controls cardiac hypertrophy. Nat Med 2007;13:613-618.

13 Small EM, Frost RJ, Olson EN: MicroRNAs add a new dimension to cardiovascular disease. Circulation 2010;121:1022-1032.

14 Su M, Chen Z, Wang C, Song L, Zou Y, Zhang L, Hui R, Wang J: Cardiac-specific overexpression of mir-222 induces heart failure and inhibits autophagy in mice. Cell Physiol Biochem2016;39:1503-1511.

15 Wu H, Huang T, Ying L, Han C, Li D, Xu Y, Zhang M, Mou S, Dong Z: MiR-155 is involved in renal ischemiareperfusion injury via direct targeting of foxo3a and regulating renal tubular cell pyroptosis. Cell Physiol Biochem2016;40:1692-1705.

16 Wang L, Zhang N, Wang Z, Ai DM, Cao ZY, Pan HP: Decreased mir-155 level in the peripheral blood of nonalcoholic fatty liver disease patients may serve as a biomarker and may influence LXR activity. Cell Physiol Biochem 2016;39:2239-2248.

17 Yang S, Li F, Jia S, Zhang K, Jiang W, Shang Y, Chang K, Deng S, Chen M: Early secreted antigen ESAT-6 of Mycobacterium Tuberculosis promotes apoptosis of macrophages via targeting the microRNA155-SOCS1 interaction. Cell Physiol Biochem 2015;35:1276-1288.

18 Yang W, Yang Y, Xia L, Yang Y, Wang F, Song M, Chen X, Liu J, Song Y, Zhao Y, Yang C: MiR-221 promotes capan-2 pancreatic ductal adenocarcinoma cells proliferation by targeting PTEN-Akt. Cell Physiol Biochem 2016;38:2366-2374.

19 Ye M, Li D, Yang J, Xie J, Yu F, Ma Y, Zhu X, Zhao J, Lv Z: MicroRNA-130a targets map3k12 to modulate diabetic endothelial progenitor cell function. Cell Physiol Biochem2015;36:712-726.

20 Ma L, Hong Y, Lu C, Chen Y, Ma C: The occurrence of cervical cancer in Uygur women in Xinjiang Uygur Autonomous Region is correlated to microRNA-146a and ethnic factor. Int J Clin Exp Pathol 2015;8:93689375. 


\section{Cellular Physiology Cell Physiol Biochem 2017;42:808-823

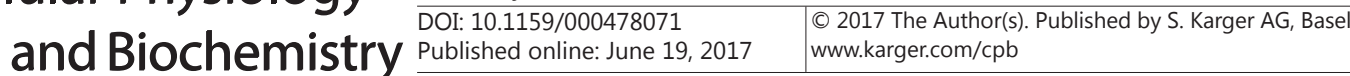 \\ Jia et al.: Potential Biomarkers for Detection of CHD}

21 Ding XQ Ge PC, Liu Z, Jia H, Chen X, An FH, Li LH, Chen ZH, Mao HW, Li ZY, Gu Y, Zhu TB, Li CJ, Wang LS, Ma WZ, Yang ZJ, Jia EZ: Interaction between microRNA expression and classical risk factors in the risk of coronary heart disease. Sci Rep 2015;5:14925.

22 Judkins MP: A percutaneous transfemoral technique. Radiology1967;89: 815-821.

23 Sun X, Zhang M, Sanagawa A, Mori C, Ito S, Iwaki S, Satoh H, Fujii S: Circulating microRNA-126 in patients with coronary artery disease: correlation with LDL cholesterol. Thromb J 2012;10:16.

24 Gensini GG: A more meaningful scoring system for determinating the severity of coronary heart disease. Am J Cardiol 1983;51: 606.

-25 Jia EZ, An FH, Chen ZH, Li LH, Mao HW, Li ZY, Liu Z, Gu Y, Zhu TB, Wang LS, Li CJ, Ma WZ, Yang ZJ: Hemoglobin A1c risk score for the prediction of coronary artery disease in subjects with angiographically diagnosed coronary atherosclerosis. Cell Physiol Biochem 2014;34:672-680.

26 Wang Y, Mao LH, Jia EZ, Li ZY, Ding XQ, Ge PC, Liu Z, Zhu TB, Wang LS, Li CJ, Ma WZ, Yang ZJ: Relationship between diagonal earlobe creases and coronary artery disease as determined via angiography. BMJ Open 2016;6:e008558

27 Hayashi M, Fujimoto K, Urushibata K, Uchikawa S, Imamura H, Kubo K: Nocturnal oxygen desaturation correlates with the severity of coronary atherosclerosis in coronary artery disease. Chest 2003;124:936941.

28 Ding XQ Ge PC, Liu Z, Jia H, Chen X, An FH, Li LH, Chen ZH, Mao HW, Li ZY, Gu Y, Zhu TB, Li CJ, Wang LS, Ma WZ, Yang ZJ, Jia EZ: Interaction between microRNA expression and classical risk factors in the risk of coronary heart disease. Sci Rep 2015;5:14925.

29 D'Alessandra Y, Carena MC, Spazzafumo L, Martinelli F, Bassetti B, Devanna P, Rubino M, Marenzi G, Colombo GI, Achilli F, Maggiolini S, Capogrossi MC, Pompilio G: Diagnostic potential of plasmatic microrna signatures in stable and unstable angina. PLoS One 2013;8:e80345.

-30 Lu HQ, Liang C, He ZQ Fan M, Wu ZG: Circulating miR-214 is associated with the severity of coronary artery disease. J Geriatr Cardiol 2013;10:34-38.

- 31 Ren J,Zhang J,Xu N,Han G,Geng Q,Song J,Li S,Zhao J,Chen H: Signature of circulating micrornas as potential biomarkers in vulnerable coronary artery disease. PLoS One2013;8:e80738.

-32 Fichtlscherer S, De Rosa S, Fox H, Schwietz T, Fischer A, Liebetrau C, Weber M, Hamm CW, Röxe T, MüllerArdogan M, Bonauer A, Zeiher AM, Dimmeler S: Circulating microRNAs in patients with coronary artery disease. Circ Res 2010;107:677-684.

33 Goettsch C, Rauner M, Pacyna N, Hempel U, Bornstein SR, Hofbauer LC:MiR-125b regulates calcification of vascular smooth muscle cells. Am J Pathol 2011;179:1594-1600.

-34 Sun YM, Lin KY, Chen YQ: Diverse functions of miR-125 family in different cell contexts.J Hematol Oncol 2013;6:6.

-35 Wang X,Ha T,Zou J,Ren D,Liu L,Zhang X,Kalbfleisch J,Gao X,Williams D,Li C:MicroRNA-125b protects against myocardial ischaemia/reperfusion injury via targeting p53-mediated apoptotic signalling and TRAF6. Cardiovasc Res 2014;102:385-395.

- 36 Ren D, Wang X, Ha T, Liu L, Kalbfleisch J, Gao X, Williams D, Li C: SR-A deficiency reduces myocardial ischemia/reperfusion injury; involvement of increased microRNA-125b expression in macrophages. Biochim Biophys Acta 2013;1832:336-346.

-37 Wang C, Yang C, Chen X, Yao B, Yang C, Zhu C, Li L, Wang J, Li X, Shao Y, Liu Y, Ji J, Zhang J, Zen K, Zhang CY, Zhang C: Altered profile of seminal plasma microRNAs in the molecular diagnosis of male infertility. Clin Chem2011;57:1722-1731.

-38 Zhang C, Wang C, Chen X, Yang C, Li K, Wang J, Dai J, Hu Z, Zhou X, Chen L, Zhang Y, Li Y, Qiu H, Xing J, Liang Z, Ren B, Yang C, Zen K, Zhang CY: Expression Profile of Micrornas in Serum: A fingerprint for esophageal squamous cell carcinoma. Clin Chem 2010;56:1871-1879.

39 Chen X, Liang H, Guan D, Wang C, Hu X, Cui L, Chen S, Zhang C, Zhang J, Zen K, Zhang CY: A combination of Let-7d, Let-7g and Let-7i serves as a stable reference for normalization of serum micrornas. PLoS One 2013;8:e79652.

40 Devaux Y, Vausort M, Goretti E, Nazarov PV, Azuaje F, Gilson G, Corsten MF, Schroen B, Lair ML, Heymans S, Wagner DR: Use of circulating microRNAs to diagnose acute myocardial infarction. Clin Chem 2012;58:559-567.

41 Schmittgen TD, Livak KJ: Analyzing real-time PCR data by the comparative C(T) method. Nat Protoc 2008;3:1101-1108. 


\section{Cellular Physiology Cell Physiol Biochem 2017;42:808-823

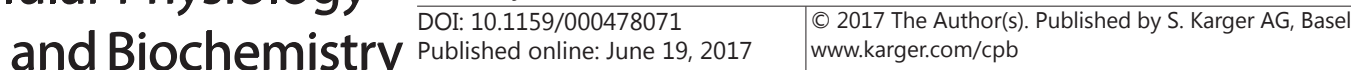

Jia et al.: Potential Biomarkers for Detection of CHD

-42 Zeller T, Keller T, Ojeda F, Reichlin T, Twerenbold R, Tzikas S, Wild PS, Reiter M, Czyz E, Lackner KJ, Munzel T, Mueller C, Blankenberg S: Assessment of microRNAs in patients with unstable angina pectoris. Eur Heart J 2014;35:2106-2114.

43 Hanley JA, McNeil BJ: The meaning and use of the area under a receiver operating characteristic (ROC) curve. Radiology 1982;143:29-36.

-44 Assman SF, Hosmer DW, Lemeshow S, Mundt KA: Confidence intervals for measures of interaction. Epidemiology 1996; 7:286-290.

45 Yang Q Khoury MJ: Evolving methods in genetic epidemiology. III. Gene-environment interaction in epidemiologic research. Epidemiol Rev 1997;19:33-43.

-46 Ling H, Fabbri M, Calin GA: MicroRNAs and other non-coding RNAs as targets for anticancer drug development. Nat Rev Drug Discov 2013;12:847-865.

47 Bartel DP: MicroRNAs: genomics, biogenesis, mechanism, and function. Cell 2004;116:281-297.

48 Navickas R, Gal D, Laucevičius A, Taparauskaitė A, Zdanytė M, Holvoet P: Identifying circulating microRNAs as biomarkers of cardiovascular disease: a systematic review. Cardiovasc Res 2016;111:322-337.

49 Wang WT, Zhao YN, Yan JX, Weng MY, Wang Y, Chen YQ, Hong SJ: Differentially expressed microRNAs in the serum of cervical squamous cell carcinoma patients before and after surgery. J Hematol Oncol 2014;7:6.

50 Cardo LF, Coto E, de Mena L, Ribacoba R, Moris G, Menéndez M, Alvarez V: Profile of microRNAs in the plasma of Parkinson's disease patients and healthy controls. J Neurol 2013;260:1420-1422.

-51 Cui M, Yue L, Fu Y, Yu W, Hou X, Zhang X: Association of microRNA-181c expression with the progression and prognosis of human gastric carcinoma. Hepatogastroenterology 2013;60:961-964.

-52 Dettmer MS, Perren A, Moch H, Komminoth P, Nikiforov YE, Nikiforova MN: Comprehensive microRNA expression profiling identifies novel markers infollicular variant of papillary thyroid carcinoma. Thyroid 2013;23:1383-1389.

53 Poliseno L, Tuccoli A, Mariani L, Evangelista M, Citti L, Woods K, Mercatanti A, Hammond S, Rainadi G: MicroRNAs modulate the angiogenic properties of HUVECs. Blood 2006;108:3068-3071.

-54 Zhang X, Mao H, Chen JY, Wen S, Li D, Ye M, Lv Z: Increased expression of microRNA-221 inhibits PAK1 in endothelial progenitor cells and impairs its function via c-Raf/MEK/ERK pathway. Biochem Biophys Res Commun 2013;431:404-408.

55 Minami Y, Satoh M, Maesawa C, Takahashi Y, Tabuchi T, Itoh T, Nakamura M: Effect of atorvastatin on microRNA 221 / 222 expression in endothelial progenitor cells obtained from patients with coronary artery disease. Eur J Clin Invest 2009;39:359-367.

-56 Pankratz F, Bemtgen X, Zeiser R, Leonhardt F, Kreuzaler S, Hilgendorf I, Smolka C, Helbing T, Hoefer I, Esser JS, Kustermann M, Moser M, Bode C, Grundmann S: MicroRNA-155 exerts cell-specific antiangiogenic but proarteriogenic effects during adaptive neovascularization. Circulation 2015;131:1575-1589.

-57 Zhu GF, Yang LX, Guo RW, Liu H, Shi YK, Ye JS, Yang ZH: MicroRNA-155 is inversely associated with severity of coronary stenotic lesions calculated by the Gensini score. Coron Artery Dis 2014;25:304-310.

-58 Chen Y, Gorski DH: Regulation of angiogenesis through a microRNA (miR-130a) that down-regulates antiangiogenic homeobox genes GAX and HOXA5. Blood 2008;111:1217-1226.

-59 Jakob P, Doerries C, Briand S, Mocharla P, Kränkel N, Besler C, Mueller M, Manes C, Templin C, Baltes C, Rudin M, Adams H, Wolfrum M, Noll G, Ruschitzka F, Lüscher TF, Landmesser U: Loss of angiomiR-126 and $130 \mathrm{a}$ in angiogenic early outgrowth cells from patients with chronic heart failure: role for impaired in vivo neovascularization and cardiac repair capacity. Circulation 2012; 126:2962-2975.

-60 Osbourne A, Calway T, Broman M, McSharry S, Earley J, Kim GH: Downregulation of connexin43 by microRNA-130a in cardiomyocytes results in cardiac arrhythmias. J Mol Cell Cardiol 2014;74:53-63.

61 Kim GH, Samant SA, Earley JU, Svensson EC: Translational control of FOG-2 expression in cardiomyocytes by microRNA-130a. PLoS One2009;4:e6161. 